\title{
Deep Psychological Learning of Japanese Anime Graphics in India Using Cognitive Computation
}

\author{
Pranjal Singh ${ }^{\mathrm{a}, 1}$, Gondi Surender Dhanunjay ${ }^{\mathrm{a}}$, Thakur Santosh ${ }^{\mathrm{b}}$, Bhavana S a, \\ K. Vengatesan ${ }^{\mathrm{c}}$ \& Abhishek Kumar ${ }^{\mathrm{a}}$ \\ ${ }^{a}$ School of Computer Science and IT, JAIN (Deemed to be University), Bangalore, India \\ ${ }^{b}$ Department of Computer Science \& Engineering, Koneru Laksmaiah Education Foundation, \\ Hyderabad, India \\ 'Department of Computer Engineering, Sanjivani College of Engineering, Kopargaon, India
}

\begin{abstract}
This paper analyzes the Japanese Animation (Anime), their art style, how it is created, about popular Anime series and movies, their growth, and adaptation in India and mainly about the growing Anime fans. Majority of Anime series and movies still use two-dimensional (2D) animation style even though there are constant technological advancements in the field of media and entertainment. Though there are setbacks in Anime, we can observe that the growth in Anime viewership is constantly rising. The animation pipeline system that is involved in the making of an Anime will also be explained in detail for a better understanding of the animation process. A research is conducted through a questionnaire form to collect the required information for the study. The data collected is examined methodically and reported. The respondents are the Anime fans, Anime viewers, Anime influencers of all age groups. The survey is mainly to understand why they prefer watching Anime, how often they watch anime? what do they like about it? how were thy influenced to watch Anime? and if Anime fans influenced other individuals to watch Anime and how many people have, they influenced to watch Anime.
\end{abstract}

Keywords. Animation, Cognitive Computing, psychological Learning, Computer Graphics

\section{Introduction}

Anime in itself has a wide reach of viewers. There are a lot of other animated films or series that use 3D animation and is developing rapidly to make fantasy visuals look much more striking and/or realistic. However, it is understood from the preliminary survey that most Anime have not reached its next stage in technology implementation, but the number of viewers and ratings are constantly growing! People have tried to capture a sense of motion in art by placing two or more images beside each other like in the cave paintings and drawings. The animation is like a smooth sequence of a set of drawn artworks which brings it to life as a single animation.

\footnotetext{
${ }^{1}$ Abhishek Kumar, School of Computer Science and IT, JAIN (Deemed to be University), Bangalore Email: abhishek.maacindia@gmail.com.
} 
When fast moving images overlap each other, it forms a deception of the images to be moving. The field of animation is now appreciated by a set of people and it has grown into industry collaborating with allied media industries, and different styles of animation are adopted for its creation.

The animation style that originated in Japan is popularly known as Anime. It is a form of entertainment which is widely accepted by viewers around the world. This study is focused on Japanese Animation (Anime) and the growing popularity of Anime and its rapid growth in the field of animation. Millennials and Generation $Z$ grew up watching Anime like Pokémon, Beyblade, Dragan Ball Z, Cardcaptor Sakura, and many more on TV without even realizing they were Japanese. Anime which wasn't initially created for kids but aimed for a wider age group of audience. Their art style, storyline and the dramatic introduction of characters challenged the animation markets and expanded the audience to various age groups; animation is highly appreciated even after continuing to use two-dimensional style.

This study is to understanding the growing popularity of Anime in the Indian context and how much people are influenced by the anime research was conducted to understand the popularity of Anime and Anime study are sparse in India.

\section{Research Methodology}

The animation style that originated in Japan and is usually associated with Japan is known as Anime. In Japanese, any form of animation from around the world is called as 'anime.' The term 'animation' is written in Japanese as ‘アニメーション' (animēshon) and in short as 'アニメ' (Anime). Starting from the 20th century when it released the earliest commercial Anime, the Japanese animation production has continued to have a steady growth. Anime is distributed widely over the internet and television broadcasts. It targets a diverse, widespread audience and also niche audiences through its various genres.

Animation in Anime: The animation is a fundamental part of an Anime. There are different types of animations like 2D animation, 3D animation, and stop motion animation. 2D animation has other techniques like hand-drawn (Traditional Animation), Rotoscope, and the vector-based 2D animation where it adopts the methods of traditional animation but is computer generated. Anime is mostly created using Traditional animation techniques. Traditional animation is where each frame is handdrawn by artists, and then the images are displayed sequentially to form animation, this is like a flipbook. Anime like Naruto and Dragon Ball $\mathrm{Z}$ have a hand-drawn background and sceneries. However, nowadays the art is done in the digital form, i.e., through the computer, as the systems have all the equipment to make it easier. But in certain circumstances, hand-drawn art is still preferred. Anime is improving its art style and animation over time, making it much better and more appealing to the audience. Most of the Anime however, utilizes limited animation, where pieces from each scene are used in the next scene if they are the same. Rather than drawing an entirely new scene every time, this process allows the animator to draw only the new details in the scene. This style of animation has its benefits; it takes lesser time to complete and costs less than other animation techniques resulting in an appealing anime that is created faster and with a lesser expense. 
Art style: Japanese animation is known for their unique art style; each Anime has its distinctive art style which is mostly unnoticeable. Creative artists draw artwork differently from each other. The art style in an anime doesn't always have to be merely appealing for it to be great, in some cases, the art style represents the Anime. For example, anime like Ergo Proxy and Psycho-pass has a dark art, which is appropriate for the dark series. Styles can change drastically in just a few years, triggered by something as small as a single movie or series. There are clear patterns that emerge when you look at the medium in chronological order. Watching how these trends repeat and develop is something fascinating. A character design or animation cut will without a doubt have its roots in from 30 or 40 years ago. It's both essential and interesting to see how everything links together in one giant web of influence. The 1960s was very much a stylistic kickstart for Anime. They saw the birth of the Anime TV series; multiple long-running series started to appear every year, which gave opportunities for directors and animators to express their own styles, this began the stylistic evolution of Anime. Certain titles started to influence each other, and distinctive trends began to emerge as more shows were made.

Anime in India: Indians are by and large winding up more intrigued by Japan as its culture impacts India. While Indians have not traditionally been affected by any East Asian culture, there are indications of developing Japanese cultural impact in India. Generally, the East Asian cultural impact has been low in South Asia. South Asia's external cultural implications in the historical setting have been to a great extent from the Middle East and the West. The average spread of Buddhism from South Asia to East Asia was to a great extent one-way and was accomplished through delegates in Central and Southeast Asia; there is no confirmation of Confucian or Taoist impact in India. Because of an assortment of chronicled and social reasons, next to no East Asian impact saturated South Asia until the nineteenth century, however, there was an exchange. Until this time, nations, for example, Korea and Japan scarcely enlisted on the Indian consciousness, if by any means.

Japanese culture has affected India more than any other East Asian culture. The explanations behind this are intricate. Indian scholarly people had appreciated Japan since the nineteenth century when Japan effectively modernized; many considered Japan to be a good example. Driving Indian learned people, for example, the Nobellaureate essayist Rabindranath Tagore kept up extensive contacts with Japanese intelligent people. Present day India and Japan have a minimal recorded history. Numerous in India sees Japan as an example of overcoming the difficulty of the East Asian model that they can securely gain from, dissimilar to China, which has disagreeable relations with India. As opposed to the talk of "civilizational ties" that occasionally underlies the Sino-Indian relationship, there is almost no civilizational learning or enthusiasm for either India or China concerning each other.

\section{Result and Analysis}

The A questionnaire was sent out in the month of November 2020 to people through social media, it was only for the Anime audience. The questionnaire was built on Google forms and sent as a link that was forwarded to many; this reduced the manual paperwork and made collecting data simpler. 155 valid responses were received, 
the respondents are Anime fans or who watch Anime. These responses form the basis of the results, analysis, and findings presented in this report. The questionnaire consisted of

- The personal information of the individual.

- How long the individual watches Anime and how many have they watched?

- Their rating of Anime to understand how much they like and what they like in Anime.

- How they were influenced and if they influence to watch Anime.

The data was analyzed, and statistics of the responses were presented in the form of graphs using Google forms, these graphs are used for better understanding and interpreting of the survey. Reference to the questionnaire is used in the paper.

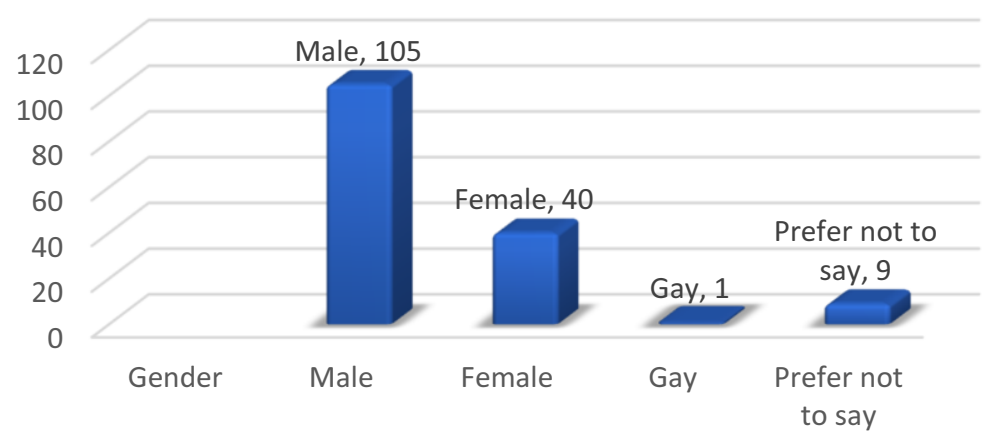

Figure 1. Viewer's Responses for Anime

The above Figure 1, shows that there are more male viewers than females who watch Anime, it is more popular among the male as there are more Shonen Anime that is popular and aired more often in India. The animated series and movies that are made in Japan have so many different genres and has so much to offer to people having different tastes, from niche community to the wide-ranging audience it has good concepts that appeal to many. Hence, people who watch Anime tend to consume it more often.

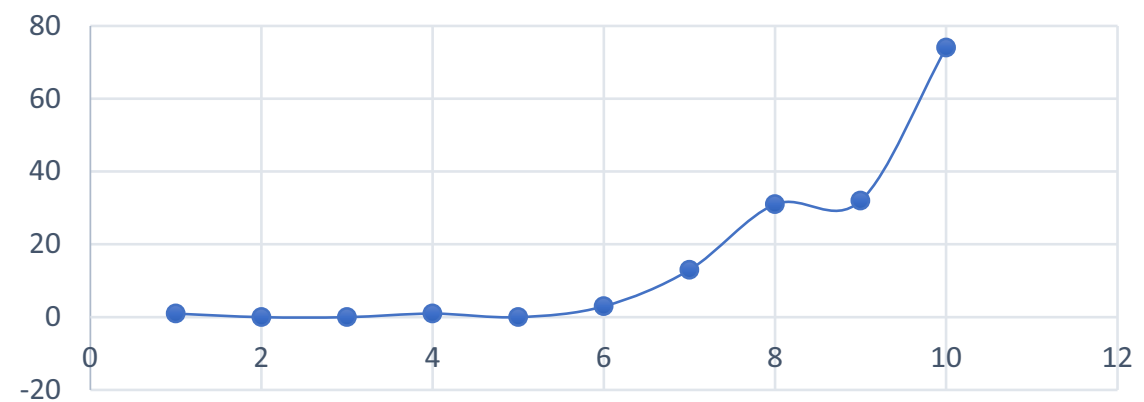

Figure 2. Rating Response of Anime 
The above Figure 2 shows rating on how many survey participants enjoy watching anime. Almost $47.7 \%$ respondents have rated 10 which says that majority enjoys watching Anime, around $20.6 \%$ have rated 9 and $20 \%$ have rated 8 . The balance population in the sample has rated from 1-6 of which $8.4 \%$ have rated it 7 and lesser than $4 \%$ have rated it lesser than 7 . This shows that the people who have watched Anime enjoy it a lot and are interested in it.

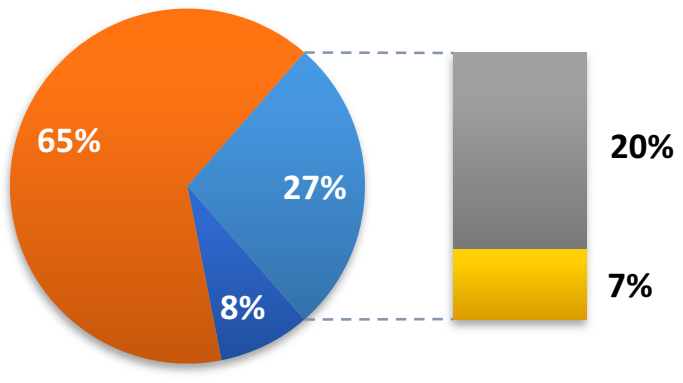

I watch only Anime

- I watch Anime and few other Animated films/series

I watch more of other animated shows more than anime

I watch both equally

Figure 3. Respondents answering how often do you watch Anime compared to other animated films

13 respondents said that they only watch Anime and no other animated shows and 100 respondents said that they watch Anime and other animated shows. So, we can observe that people who have watched or watch Anime prefer watching more of Anime over other animated films. There are 31 people who watch more of other animated shows more than Anime and rest 11 have said that they watch both equally.

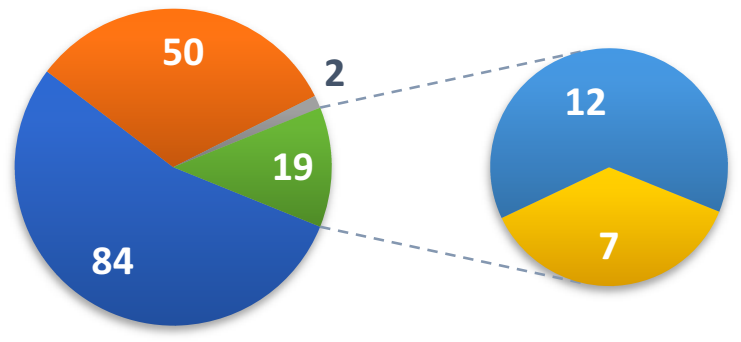

By other people/person's influence $\mathbf{a}$ Television

Advertisements Games

Other sources

Figure 4. Ways of participants are influenced to watch anime 
From the above Figure 4 we can clearly observe that Anime is being popularized mostly by other people's influence and secondly by television. There are a lot of Anime series like 'Pokemon', 'Shinchan' and 'Doraemon' that are telecasted in Indian cartoon channels. till 2017 TV Channel named ANIMAX was broadcasting in India where many viewers were influenced to watch Anime in the channel. even though ANIMAX stopped broadcasting in India Fans Now majorly watch on the web. Only a very few audiences were influenced by advertisements, games and other means as mentioned by the participants.

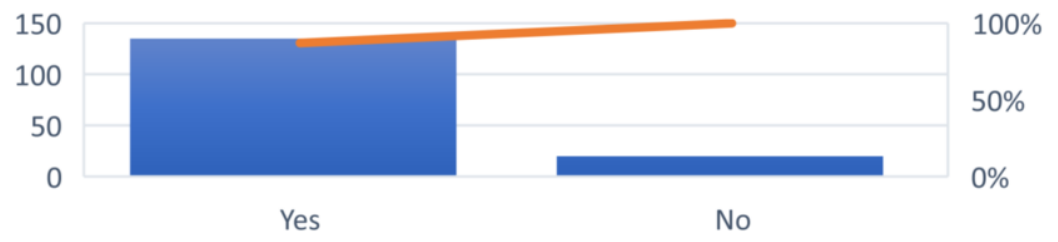

Figure 5. People influence others to watch Anime.

As seen in the above Figure 5 most people who watch Anime recommend and influence others to watch too. As we can see $87 \%$ of participants who watch Anime have agreed that they influence others to watch Anime and around $12 \%$ said that they do not influence others.
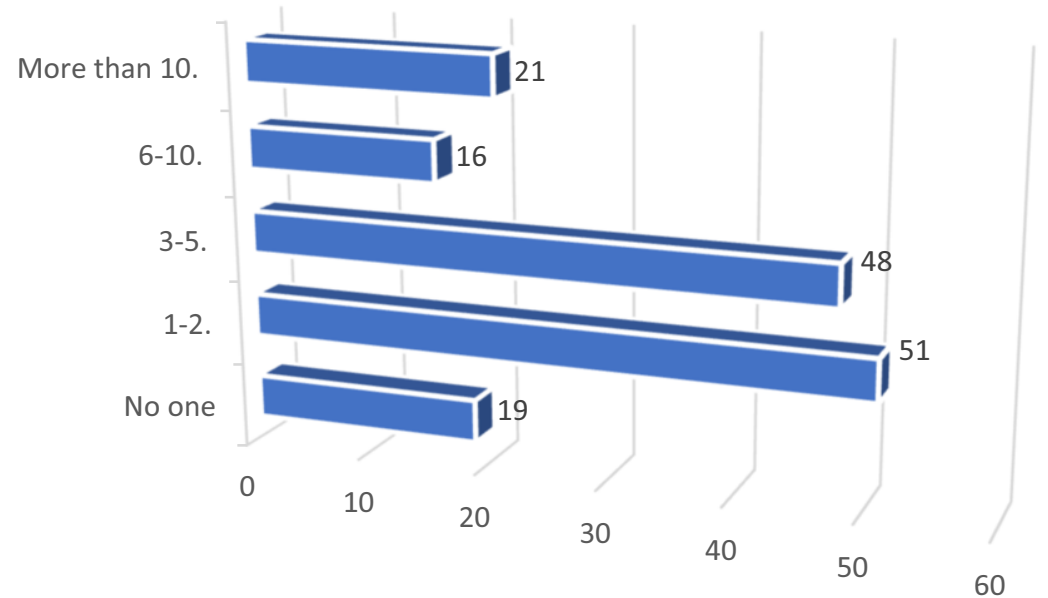

- Series 1

Figure 6. Peers Pressure Analysis

As seen in the above Figure 6 most of the people who watched Anime have influenced at least one other person to watch Anime. Only around 12\% have not persuaded others to watch Anime. Around 30\% people have influenced about $1-2$ and the same percent have influenced about $3-5$. The others have influenced more than 5 people to watch Anime. 


\section{Conclusion and Future discussion}

Anime is an animation style that originated in Japan and is usually associated with it. The production of Anime started back in the 20th century and continued to grow steadily. Anime mostly uses the Traditional animation method. Initially, each frame and background of the Anime was hand-drawn, and as technology developed, Anime also evolved, now digital art is used to create beautiful artwork to bring realism in Anime. Anime has its animation techniques like the 'Obari Punch,' 'Itano Circle,' and the 'Yutapon Cubes' that are used by animators in the creation of many shows; these techniques have also evolved making them more appealing to the audience. Anime has a unique art style; unlike the other animations we see. Anime has various genres that reach a diverse range of audience. The Japanese culture has had a significant impact on the Indian culture in many ways. We have adapted and learn from them and their culture. Anime is one of the things that has widely impacted India and its people. The youth of India are engaged in activities related to Anime and conventions and actively participate in them. The Japanese government has also come forward to help spread its culture and in turn, improve the relationship between the two countries. As anime is gaining more and more popularity, it is influencing the people to get involved in it and grow as a community.

The research to understand 'The Anime Evaluation' had 155 responses, most of these responses were from Anime fans and the others who watch Anime. The responses showed that there were more male viewers than the others. Also, most of them watch Anime regularly, and only a few watches it rarely. Most of the participants have been watching Anime for more than 3 years and have watched quite a lot of Anime shows since they started. More than $88 \%$ participants have rated Anime more than 8 . We can also conclude that most people like the 2-dimensional animation style that most Anime use. A lot of Anime fans influence other people wo watch Anime, and they have started watching Anime by their influence and they themselves have been influenced mostly by other people to watch Anime. According to this survey we can confidently conclude that Anime is mostly popularized by Anime fans.

\section{References}

[1] Poitras, Gilles (2000). Anime Essentials: Every Thing a Fan Needs to Know. Stone Bridge Press.

[2] Poitras, Gilles (1998). Anime Companion. Berkeley, California: Stone Bridge Press.

[3] Potharaju, S. P., \& Sreedevi, M. (2018). A novel cluster of quarter feature selection based on symmetrical uncertainty. Gazi University Journal of Science, 31(2), 456-470..

[4] Kumar, A., Vengatesan, K., Rajesh, M., \& Singhal, A. (2019). Teaching literacy through animation \&amp; multimedia. International Journal of Innovative Technology and Exploring Engineering, (5), 73-76.

[5] A. Kumar, K. Vengatesan, A. Singhal and D. K. Verma, "3D Lighting Courseware development for 3D Motion Picture Science," 2018 International Conference on Recent Innovations in Electrical, Electronics \& Communication Engineering (ICRIEECE), 2018, pp. 2321-2323, doi: 10.1109/ ICRIEECE44171.2018.9009258.

[6] Kumar, A. Singhal, K. Vengatesan and D. K. Verma, "Study and Research of 3D Animation Courseware Development," 2018 International Conference on Recent Innovations in Electrical, Electronics \& Communication Engineering (ICRIEECE), 2018, pp. 2514-2516, doi: 10.1109/ ICRIEECE44171.2018.9008670.

[7] J. Adam Jones, J. Edward Swan II, Gurjot Singh, Josh Franck, and Stephen R. Ellis. The Effects of Continued Exposure to Medium Field Augmented and Virtual Reality on the Perception of Egocentric 
Depth. In Poster Compendium, Proceedings of ACM SIGGRAPH Symposium on Applied Perception in Graphics and Visualization (APGV 2009), pp. 138, September 2009. DOI: 10.1145/ 1620993.1621032

[8] Anna, A. (2007). The social implications of enjoyment of different types of music, movies, and television programming. Western Journal of Communication, 71(4), 259-271. 